\title{
An approach of inertia compensation based on electromagnetic induction in brake test
}

\author{
Xiaowen Li \\ College of Computer Science and Technology \\ Chongqing University of \\ Posts and Telecommunications \\ Chongqing, China
}

\author{
Han Que \\ College of Computer Science and Technology \\ Chongqing University of \\ Posts and Telecommunications \\ Chongqing, China
}

\begin{abstract}
This paper briefly introduced the operational principle of the brake test bench, and points out the shortcomings when controlling the current of brake test, which means the reference measuring data is instantaneous. Aimed at this deficiency, a current control model based on electromagnetic induction and DC voltage is proposed. On the principle of electromagnetic induction, continuous data and automatic processes are realized. It significantly minimized errors owing to instantaneous data, and maximized the accuracy of the brake test.
\end{abstract} former

Keywords-Brake test; Electromagnetic induction; DC trans-

\section{INTRODUCTION}

Vehicle brake design is one of the most important processes in the vehicle design. In order to detect the comprehensive performance of brake, thousands of brake tests are required. The actual test is generally divided into the road test and simulation test. The simulation test basically based on vehicle brake test bench. However, the road test is impossible to operate during vehicle design stage. Thus, brake test simulation on brake test bench is the best method in this situation. The principle of this method is simulating the road test on the brake test bench as much as possible.

In the simulated road test experiments of brake test for brake performance, due to the mechanical inertia flywheel group could not concisely achieve the rotational inertia which test system required, typically the industry introduce motor into the test bench. In order to meet the principles of simulation tests, the current of the motor could be controlled specifically when it participating in the experiments to compensate the energy the mechanical inertia required. However, due to the complexity of the brake performance, the precise relationship between the motor driving current and the time is difficult to obtain. The normal method is discretization. The entire braking time is discretized into quite a few tiny time periods. Then according to the instantaneous speed and instantaneous torque which observed in previous period, devising the driving current value of the current time. This process successively operated until the completion of brake test. Actually, the driving current value devised by this discretion method possess a certain error comparing to theory value. The error would cause unnecessary trouble in the brake test. Through the establishment of the current control and the DC transformer model based on electromagnetic induction and electromagnetic induction method, the error is able to be eliminated. Consequently, improvement of the brake test bench experiments is realized.

\section{COMPONENTS OF TEST BENCH SYSTEM}

1) Basic components of brake test bench: Brake test bench generally consists of spindle with flywheel group, motor which driving the spindle rotation, basement, assist devices applying on the brake, measurement system and control system, etc. Shown in Figure 1.

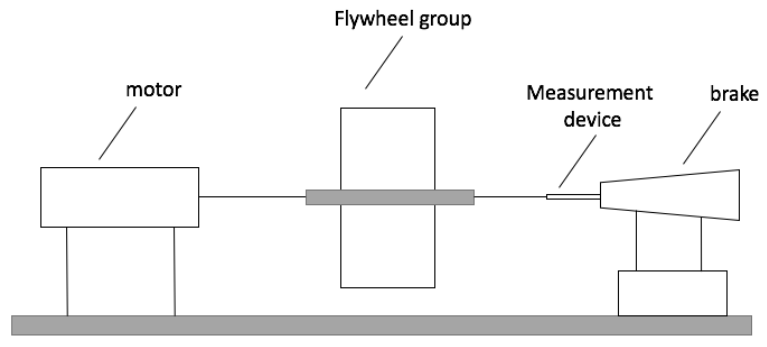

Fig. 1: Basic components of brake test bench

As shown, when the brake test start operating, the motor drives the flywheel group rotating to reach the set rotational speed which corresponding to the speed of vehicle (in the simulation, assuming the angular velocity of the spindle is always constant with the angular velocity of the wheels). Once it reached the set speed, then disconnecting the power supply. Meanwhile, apply braking for the motor. When the termination condition is satisfied, one brake test is completed. Comparing to brake test bench, the vehicle wheels in road test would absorb load when applying brake. Thus, the energy which the 'load' posses when it moving with the vehicle (ignoring rotation energy of the wheel itself) should be equally converted to the energy of flywheel group, spindle and other devices in brake test bench when they are rotating. This energy which corresponding to the rotational inertia (short for inertia below) is called equivalent rotational inertia. In order to simulate the road test with mechanical inertia accurately, we introduce the motor into the brake simulation test and controlling the current of motor with regular rules to compensate the energy which mechanical inertia require. Consequently, the principles of simulation test are satisfied. Basic assumptions: 
1. Assuming that flywheel group is strictly rigid.

2. Assuming that the braking torque of road test provided entirely by the brake.

3. Assuming that the brake torque from motor and brake is applying on the spindle completely.

4. Assuming that brake torque of spindle is entirely provided by the brake

5. Assuming that the angular velocity of spindle in brake test bench is constant with the angular velocity of the wheel in road test.

6. Assuming that the measured data is accurate and reliable.

7. Assuming that when the measurement is performed, the time interval is stable after discretization.

In the experimental test, the entire simulation processes are shown as below:

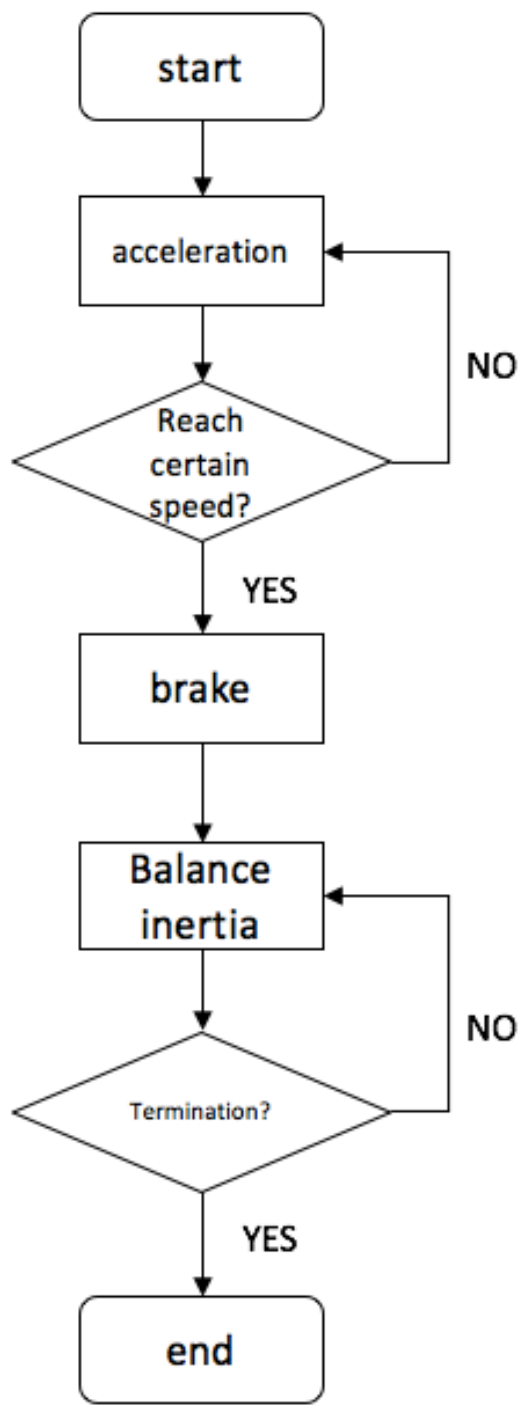

Fig. 2: Simulation process

In a typical test bench experiment, the motor driving current control is determined by measuring the discrete instan- taneous speed and instantaneous torque. Therefore, the errors are larger in actual situation. According to the thought of continuous discrete control, we introduce the current control and the DC transformer model based on electromagnetic induction.

2) Establish the current control model based on electromagnetic induction and DC transformer: On the basis of brake test bench system, a miniature DC generator is connected and fixed on the spindle. Accordingly, the generator coil inside the generator have the same rotational speed with the spindle. Thus, when the spindle is rotating, the DC generator will produce induced electromotive force by electromagnetic induction.

We introduce induced electromotive force into the circuit 1 . By changing the current $I_{1}$, the inductance $\mathrm{L}$ in circuit 1 generate induced electromotive force. The circuit 2 generate induced electromotive force through transformer inductance L. We add a DC transformer in circuit 2 and connect the power supply to provide additional energy output. By adjusting the magnification of the voltage, making the voltage applied to both ends of the dynamic system in the brake test bench. Meanwhile, the energy of this voltage provided by the electric motor is equal to the energy consumed by heat plus the energy required for spindle rotation. Thus, we can make the discrete data which in original model continuous and reduce random errors generated by discretization.

The main structure and a circuit diagram of the model shown in Figure 3:

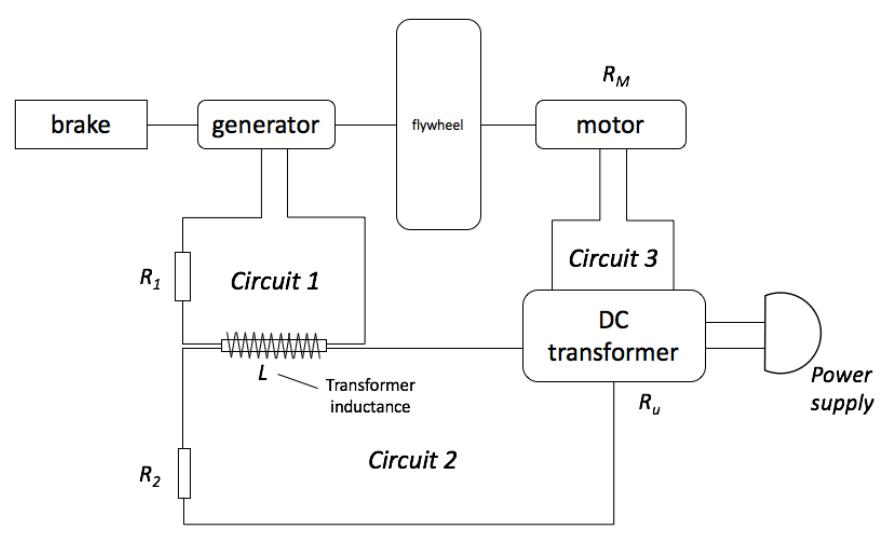

Fig. 3: Circuit diagram of the model

3) DC generator: The following context described the DC generator.

Figure 4 is a simple DC generator model. The $N$ and $S$ are a pair of fixed poles. They are either permanent magnet or electromagnet. There is a rotatable iron cylinder between the magnetic poles which known as the armature core. On the surface of the core fixed the armature coil ' $a b c d$ ' which consist of insulating conductors. On both ends of the coil connected to 2 mutually insulated arc-shaped copper respectively. The arcshaped copper is called commutator segments. The combination of them is called a commutator. The commutator brushes $\mathrm{A}$ and $\mathrm{B}$ are placed on the commutator stationary and sliding contact with the commutator. The coil ' $a b c d$ ' connected to external circuit through the commutator and brushes. The armature core, armature coils and commutator together integrally 
called an armature. The armature rotating with prime mover and transform the mechanical energy into electrical energy and supplied to the electric load which connected to the brushes.

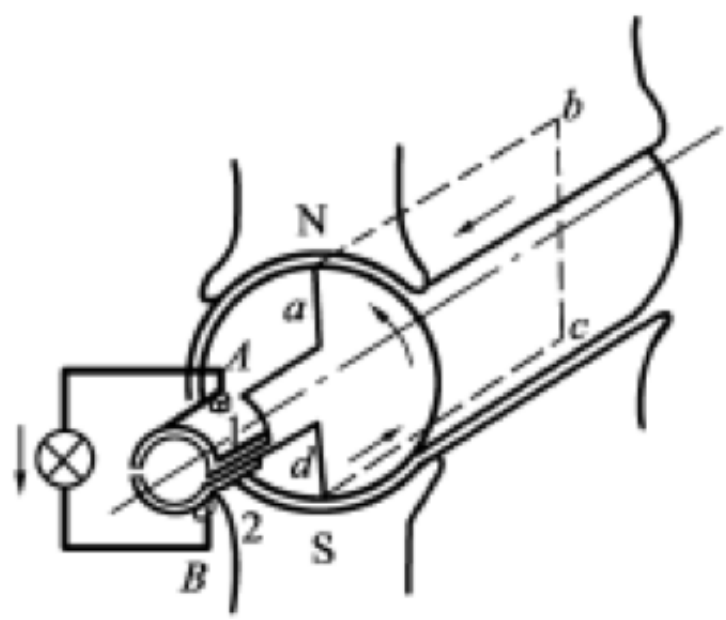

Fig. 4: DC generator model

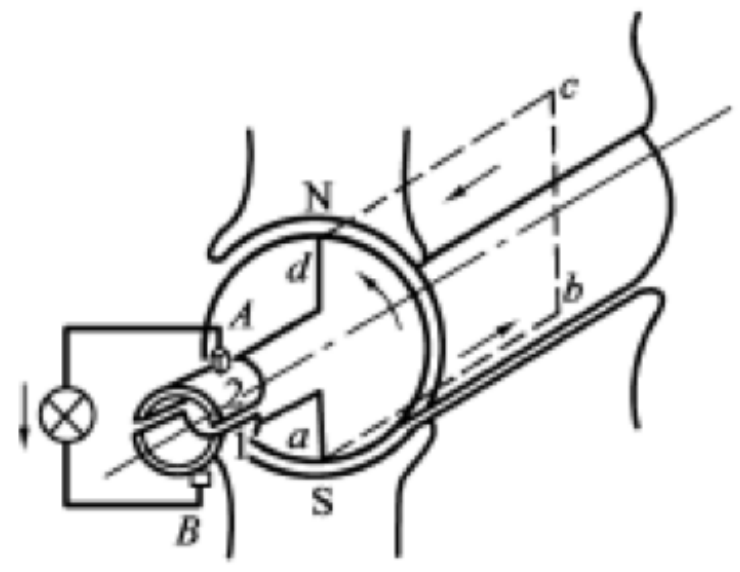

Fig. 5: DC generator model

In figure 4, when the armature counterclockwise rotating, the coil edges $a b$ and $c d$ cut magnetic lines and generate induced electromotive force. The direction of induced electromotive force shown in figure 4 is from dcba. The brush $A$ is positive and brush $B$ is negative. The direction of current flow in the external circuit is start from positive $A$ to the negative $B$ through circuit load which could be judged according to the right-handed rule.

When the armature rotated with 180 degrees, as shown in figure 5, the induced electromotive force direction of the coil changed to $a b c d$. The brush $A$ originally contact with the commutator 1 consequently changed to contact with the commutator 2 . The brush $B$ originally contact with the commutator 2 consequently changed to contact with the commutator 1 . Thus, the brush $A$ still positive and brush $B$ is still negative. The analysis shown above turns out that when a certain edge of the coil rotating from the range of magnetic poles to the range of the adjacent opposite magnetic poles, the direction of induced electromotive force changed for one time. However, in terms of the external circuit, the direction of the induced electromotive force between the brushes is constant and the value of induced electromotive force ranges from zero to the maximum.

Although we can resolve induced electromotive force and current in a constant direction through this kind of single coil DC generator, but its value is pulsating. This is the basic principle of DC generator.

Actually, the value of induced electromotive force generated by the DC generator is not only pulsating but volatile. Thus, the electromotive force is not acceptable with actual requirements. To reduce the fluctuation of the electromotive force, we can appropriately increase the number of coils and the armature segments. As an example, winding two coils on the armature, namely there is one coil edge every 90 degrees. Thus, halfwave induced electromotive force is changed to 90 degrees and the pulsating has been significantly reduced. The coil number in practical applied DC generator is generally multiple and the number of magnetic pole pairs is also more than one pair. Thus, the fluctuation of induced electromotive force is rather slight (experiments and analysis show that when the number of conductors in each magnetic pole is more than 8 , the fluctuation of induced electromotive force is less than 1\%). Thus, we considered it as constant DC electromotive force.

4) DC adapter: Firstly, transforming direct current boost voltage of direct current into alternating through electronic components and then change the voltage through the transformer. This process is used for inverter voltage. The devices used for converting DC power boosting called an inverter. Transformer works based on electromagnetic induction principle. Transformer owns two sets of coils, primary coils and secondary coils. The primary coils are outside of the secondary coils. When the alternating current flow through the primary coils, the transformer cores generate alternating magnetic field and the secondary coils generate induced electromotive force. The ratio of transformer coil turns is equal to the voltage ratio. As an example: the primary coil is 500 turns and the secondary coil is 250 turns. If the voltage of primary coils applied on $220 \mathrm{~V}$ (alternating current) then the voltage of secondary coils is $110 \mathrm{~V}$. Transformer can either boost voltage or step-down voltage. If the turns of primary coils are less than the turns of secondary coils, namely it is a boost transformer which promoting to a high voltage.

Generally, the DC voltage transformer is used for the conversion of voltage and obtain energy from a certain power supply. Then use oscillators turning the current to alternating current and use the transformer to boost or drop voltage. Finally, reduced the current to the direct current through a rectifier circuit. The feedback loop control circuit is necessary if high criterial required in obtaining relatively stable output voltage.

\section{MODEL SOLUTION}

First, we establish a mathematical model which the motor driving current depends on observables. The model is based on the rigid physics theory and other physics theories. According to the force analysis of any rigid objects and relevant rigid physics conclusions, the torque of rigid objects is proportional to the product of its rotational inertia and angular acceleration, namely: 


$$
M \propto J \beta
$$

When the torque, rotational inertia and angular acceleration are all SI units, the proportional coefficient is 1 . We can resolve the relationship of rigid torque, rotational inertia and angular acceleration, namely:

$$
M=J \beta
$$

Based on the conditions we assumed, the angular velocity of the spindle in the brake bench test is completely constant with the angular velocity of the wheel in road test. And the equivalent rotational inertia $J_{0}$ and the mechanical inertia $J_{m}$ are both constant value.

We assume that the wheel speed is uniformly changed when the car start braking in the road test. Namely, the wheel angular velocity decrease linearly and angular acceleration remain constant. Thus:

$$
\beta=\frac{\Delta \omega}{\Delta t}
$$

Due to the torque is provided by the brake completely in road test. The torque of brake denoted as $M_{b}$ is a constant value according to the relationship of torque, rotational inertia and angular acceleration, which is:

$$
M_{b}=J_{r} \beta
$$

The time curve of torque, rotational inertia and angular acceleration are shown as below:

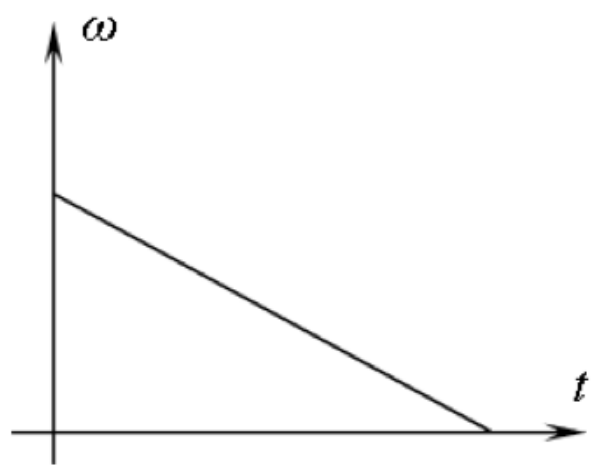

Fig. 6: Time curve of angular velocity

In the simulation test, the flywheel angular velocity decreases linearly and angular acceleration remains constant. It is the same with the assumptions in the road test. Due to the mechanical inertia is less than the equivalent rotational inertia, the angular acceleration of the flywheel in simulation test is greater than the angular acceleration of wheel in road test. Therefore, the driving current is provided to compensate the torque gap caused by the insufficient rotational inertia. Thus:

$$
M_{c}=M_{b}-M_{m}=\left(J_{r}-J_{m}\right) \cdot \beta=\left(J_{r}-J_{m}\right) \cdot \frac{\Delta \omega}{\Delta t}
$$

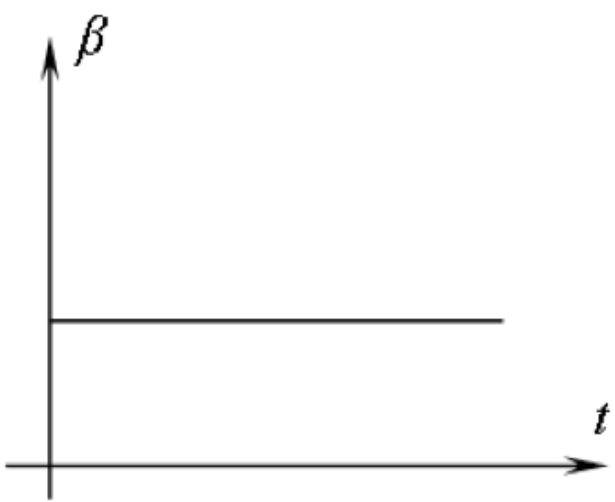

Fig. 7: Time curve of angular acceleration

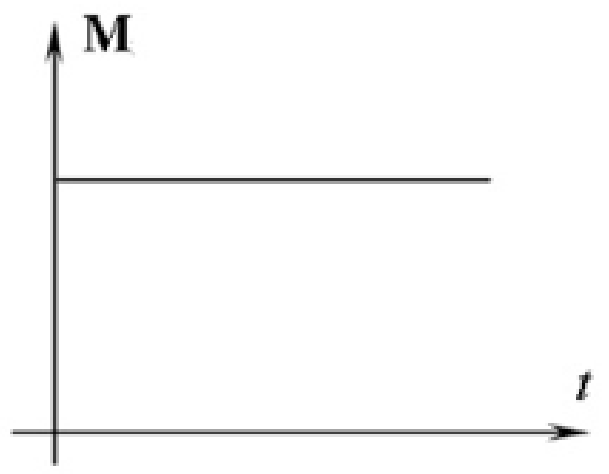

Fig. 8: Time curve of torque

According to the motor parameters, the motor driving current is proportional to its torque and we denoted the proportional coefficient as $\theta$ :

$$
I=\theta \cdot M_{m}
$$

Therefore, according to the conclusions above, we established the motor drive current mathematical model depending on the observables, namely:

$$
I=\theta \cdot\left(J_{r}-J_{m}\right) \cdot \frac{\Delta \omega}{\Delta t}
$$

In Figure 3, suppose that the equivalent magnetic induction density of the coil in the generator is $B$. The equivalent number of coil turns is $n$. The magnetic flux area of the coil is $S$. The rotational angular velocity of coil is $\omega$ and consistent with the spindle rotational speed. According to Faraday's law of electromagnetic induction, induced electromotive force generated by the DC generator is:

$$
E_{1}=2 n B S \frac{d \varphi}{d t}=2 n B S \omega
$$

Introducing the current generated by DC generator into the circuit 1 when the coil is rotating, in which the self-inductance coefficient of the inductance is $L$ and the resistance of the 
closed circuit is $R_{1}$. According to Ohm law, the induced current in circuit 1 is:

$$
I_{1}=\frac{2 n B S \omega}{R_{1}}
$$

Then transfer the changing current from circuit 1 into the circuit 2 through the transformer inductance. Namely the circuit 2 generate the induced electromotive force by the changing current from circuit 1 . In this situation, the transformer inductance efficiency is $\eta$. The inductance inductance coefficient in circuit 2 is $L$. The resistance in closed loop circuit 2 is $\left(R_{2}+R_{u}\right)$. Meanwhile, the induced electromotive force in circuit 2 equal to the product of current change rate and self inductance in circuit 1 . Therefore, the induced electromotive force in circuit 2 is:

$$
E_{2}=\eta L \cdot \frac{d I_{1}}{d t}=\frac{2 \eta n L B S}{R_{1}} \cdot \frac{d \omega}{d t}
$$

The relationship between the spindle angular acceleration and angular velocity is:

$$
d \omega=\beta \cdot d t
$$

Therefore, the induced electromotive force in circuit 2 can be written as:

$$
E_{2}=\frac{2 \eta n L B S}{R_{1}} \cdot \beta
$$

As shown in figure 3, Suppose that the resistance connected to the DC transformer in circuit 2 is $R_{u}$. According to the principle of voltage dividing, the voltage transporting to the $\mathrm{DC}$ voltage transformer is:

$$
E_{u}=E_{2} \cdot \frac{R_{u}}{R_{2}+R_{u}}=\frac{2 \eta n L B S R_{u}}{R_{1}\left(R_{2}+R_{u}\right)} \cdot \beta
$$

In circuit 3, we denoted the current which the motor required for providing torque as $I_{3}$, according to the the relationship among the current, inertia compensation and the rotational angular velocity, the value of $I_{3}$ can be obtained:

$$
I_{3}=k \cdot\left(J_{r}-J_{m}\right) \cdot \frac{d \omega}{d t}=k \cdot J_{c} \cdot \beta
$$

Let motor internal resistance be $R_{M}$. The coefficient proportion of energy for self-consumption to the total energy in the entire circuit 3 is $\xi$. Then we can solve the electromotive force which circuit 3 required, which denoted as $E_{3}$. These are the equations:

$$
\int E_{3} I_{3} d t=\frac{1}{\xi} \int I_{3}^{2} R_{M} d t
$$

then:

$$
E_{3}=\frac{k \cdot J_{c} \cdot \beta \cdot R_{M}}{\xi}
$$

Simultaneous equations:

$$
E_{u}=\frac{2 \eta n L B S R_{u}}{R_{1}\left(R_{2}+R_{u}\right)} \cdot \beta
$$

DC transformer voltage ratio is obtained as follow:

$$
\lambda=\frac{E_{3}}{E_{u}}=\frac{k \cdot J_{c} \cdot R_{M} \cdot R_{1}\left(R_{2}+R_{u}\right)}{2 \eta \xi n L B S R_{u}}
$$

When the test bench experiments come to 'Start brake' step shown in the flowchart and disconnect the power switch which spin up the flywheel, then connect the motor with the entire circuit which designed as figure 3(including power supply energy for DC transformer). The rest processes of brake test would be completed automatically.

\section{ERROR ANALYSIS}

According to the computer simulation model above, we can start the error analysis based on the data and obtain the time curve of energy relative error, shown in figure 9:

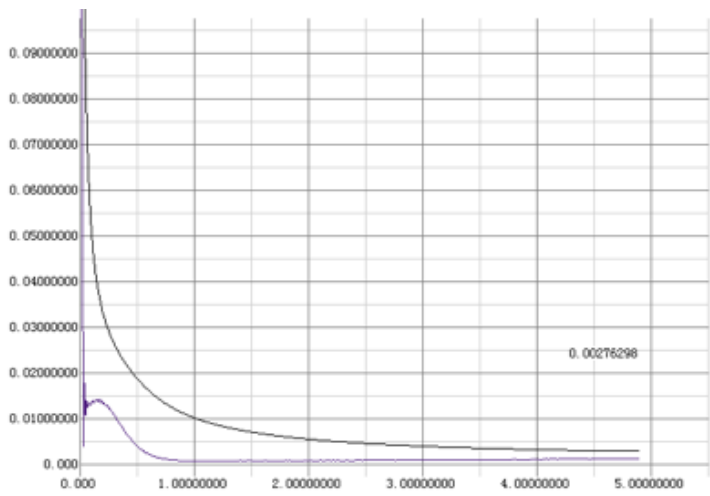

Fig. 9: Error analysis

Based on energy relative error curve, the error can be controlled within $1.5 \%$ in the beginning of the experiment. The error in this part is mainly systematic error and error due to weakly mutual inductance generated between the circuit 1 and the circuit 2 . After $0.9 \mathrm{~s}$, the error of the entire system is only $0.1 \%$. The average energy relative error of the entire process is $0.276 \%$. Apparently, the error with this model is rather slight.

\section{CONCLUSION}

The model is strongly supported by software. Especially the support from physics, mathematics and relevant theory, and computer control. Due to the motor drive current is generated automatically based on electromagnetic through physical theory and the motion state of the entire test bench system. Therefore, the result is more precise than the PID intelligent control technology in industry which has been widely used 
currently. This is the advantage of this model.

However, the model needs to be supported by precise hardware, as well as the various hardware parameters. For instance, the various parameters of the motor must be very precise. In summary, the error of the model mainly from system hardware errors, which is insufficient for this model.

\section{REFERENCES}

[1] H. Kopka and P. W. Daly, A Guide to $B T_{E} X$, 3rd ed. Harlow, England: Addison-Wesley, 1999.

[2] Gezhi Zhu and Xiaolan Zhang, Motor and Drag. Chongqing University Press,Sep 1999.

[3] Renguang Wang, Zhaodu Liu, Yuefeng Ma, Zhiquan QI, Improved design of brake test bench. Agricultural Machinery, Vol.37, No.6, May 2006.

[4] Jianjun Chen, Brake test bench mechanical inertia electrical analog control method. Shaoxing, Zhejiang Province, Shaoxing University Institute, April 2007

[5] Shubao Wei, Changsheng Xu, Inertial load brake test bench design and virtual prototype simulation analysis. Wuhan University of Technology, Vol.30, No.6, pp. 988, Dec 2006.

[6] Feng Xie, Jiwang Fang, Juguang Lin, Single-ended inertia brake performance test bench development. CLC: TH16; U467. Technology and Equipment, Nov 2008.

[7] Bo Liang, Yuren Li, Fuzzy self-tuning PID application of inertia simulation in the brake test bench. Electronic Measurement Technology, Vol. 31, No.10, October 2008

[8] Haishu Du, Zhi Yang, Rongsheng Qiu, Neural intelligent PID control algorithm. CLC: TP273.2, Document code: A, Jan 1999.

[9] Shiqin You, Flywheel rotational inertia calculation. Huainan Technical College, Volume 2 (Total 5), Apr 2002

[10] Qiang Fei, Wuyun Zhao, Fei Dai, Design of vehicle brake speed control system based on LabVIEW [J]. agricultural equipment and vehicle engineering, 2013, 51 (11): 6-10.

[11] Qiang Song, Huihui Zhang, Yiming Feng, Research on grey prediction control of brake test bench[J]. [1] Earth, 2014 (5).

[12] Tonghua Niu, Hu Ni, Development of Automotive Hydraulic brake test bench $[J]$. Quality of goods and construction and development, 2015 (1) 